\title{
Self-repairing of polymer-cement concrete
}

\author{
P. ŁUKOWSKI* and G. ADAMCZEWSKI \\ Faculty of Civil Engineering, Warsaw University of Technology, 16 Armii Ludowej Ave., 00-637 Warszawa, Poland
}

\begin{abstract}
Self-repairing means ability to the total or partial recovering of the properties which were worsened as a consequence of damage of the material. The subject of the paper is evaluation of ability to self-repair of a cement composite modified with epoxy resin without a hardener. The methodology of investigation of self-repairing building materials, developed by the authors, has been described, with controllable enforcing of the limited weakening of the material. Also, the self-repair degree has been defined as the measure of self-repairing ability of the building composites. The material model of the epoxy-cement composite has been developed on the basis of the tests results. The material optimization of the composite towards the maximum self-repairing ability has also been carried out. The results of investigation have confirmed the possibility of self-repairing of the cement composite modified with the epoxy resin without hardener. The conclusion and further research needs in the range of the self-repairing epoxy-cement composites have been pointed out.
\end{abstract}

Key words: epoxy-cement composite, microcracks, modification, self-repairing.

\section{Introduction}

Repairs of building structures are often difficult and expensive, however, they are also inevitable. One of the main tasks of today's building materials engineering is development of the more durable materials, the use of which should diminish the frequency of necessary repairs. Another challenge is searching for materials, which could make the repairing process itself easier. The prospect of merging these both research directions is created by the concept of self-repairing concretes and mortars.

The problem, which has to be solved during the repair of the concrete structure, is how to advance with the repair material to the proper place - where the damage has occurred. This is usually less complicated in the case of the surface repairs, as the access to the repaired surface is relatively easy. The problem is created by damages of concrete inside the building element. There are microcracks which occurred inside the concrete, particularly in the interfacial zones between the cement paste and the aggregate; the microcracks expand and form the network, which decreases the concrete strength and makes the ingress of the aggressive substances inside the concrete much easier. This, in turn, leads to concrete corrosion and destruction. Placing the repair material into the crack is possible only after the damage becomes visible, what often takes place when damage is quite serious. The method of repair of cracks is usually injection [1]; the method, which is rather complicated and expensive as well as requires the skilled staff for proper execution. The injection is also connected to a large degree of uncertainty of its effectiveness [2]. The alternative solution may be making the material able to repair its damages without necessity of external intervention. The concept of self-repairing consists in the assumption that the repair material can be placed inside the concrete during production of the concrete mix, i.e. well before the damage is occurring. When the internal stresses in the concrete exceed the specified level, the repair material is activated. The methods of self-repairing differ from each other, first of all, by the mechanism of this activation. Various possibilities of self-repairing have been briefly described in the paper. The particular attention has been paid to the use of epoxy resin without hardener, which is an idea initiated and first investigated by Y. Ohama [3], now developed by the authors [4].

\section{Self-healing and self-repairing}

Self-healing of concrete means the process leading to the partial recovery of the damaged material properties, which is not controlled and independent on the designer [5]. The concrete carbonation, delayed cement hydration and the phenomena taking place during water flow through the concrete, can be considered as such processes.

The carbonation is the process in which calcium hydroxide present in the hardened concrete reacts, in the presence of moisture, with the atmospheric carbon dioxide:

$$
\begin{gathered}
\mathrm{CO}_{2}+\mathrm{H}_{2} \mathrm{O} \rightarrow \mathrm{H}_{2} \mathrm{CO}_{3} \\
\mathrm{H}_{2} \mathrm{CO}_{3}+\mathrm{Ca}(\mathrm{OH})_{2} \rightarrow \mathrm{CaCO}_{3}+2 \mathrm{H}_{2} \mathrm{O}
\end{gathered}
$$

The produced calcium carbonate has larger volume than the reactants and is less soluble than calcium hydroxide. As a consequence, the concrete becomes less porous, which leads to increase of strength and limitation of ingress of the aggressive chemicals. The negative result of carbonation is lowering of alkalinity of concrete and loss of protection ability of concrete in regard to the reinforcing steel [6].

The delayed, "residual" hydration of cement may result in the partial recovery of the mechanical properties and tightness of the cracked concrete [7]. The potential source of the repair material is non-hydrated cement, usually present in some

*e-mail: p.lukowski@il.pw.edu.pl 
amount in the hardened concrete. The cracks inside the concrete might be filled with the products of cement hydration [8, 9] (Fig. 1). a)

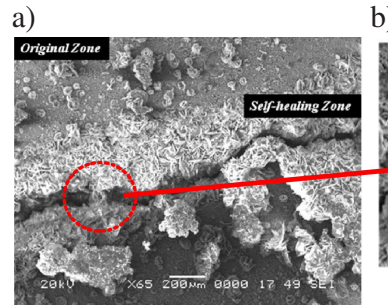

b)

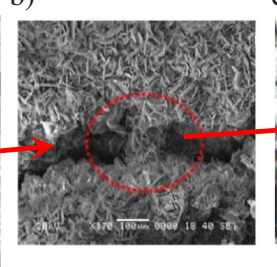

c)

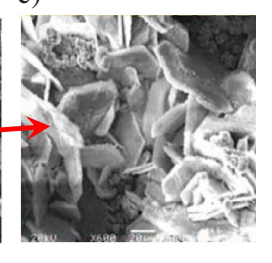

Fig. 1. Self-healing of concrete as a result of delayed cement hydration: a) cracked microstructure of the concrete, b) filling of the microcrack, c) products of cement hydration inside the selaed microcrack after Ref. 9

The effect of such self-healing is possible only in the presence of water. The multiple cycles of saturation and drying might also support the tigthening of the cracked concrete [10], mainly due to the re-crystallisation of calcium carbonate inside the crack [11]. The self-healing can be a result of precipitation of some deposits from water $[12,13]$ as well. However, the saturation of concrete with water is definitely harmful for the material.

The effectiveness of concrete self-healing is connected with the width of the cracks. V.C. Li and E.H. Yang have developed the cement composite, in which this width is controlled by using steel fibres, thus improving the self-healing efficiency; the material is called Engineered Cementitious Composite [14, 15].

Self-repairing means ability of a material to the total or partial recovering of its properties, worsened as a consequence of damage, without any external intervention. The self-repair should proceed in the place and time expected by the designer, i.e. where and when it is desirable. However, achieving such ability is not easy.

\section{Methods of implementation of self-repairing ability to concrete}

One of the methods of implementation of self-repairing ability to concrete is the use of microorganisms [16]. The promising results were obtained using Bacillus Pasteurii. The product of their metabolism is calcite $\mathrm{CaCO}_{3}$, which can be deposited in the cracks. However, the relatively effective action of the bacteria is limited to the narrow range of its concentration in the concrete. Also, the specific chemical conditions are necessary (constant $\mathrm{pH}$, moisture, etc.) and the bacteria considered are aerobs, thus the oxygen access is necessary.

The other way is the concept of dispersed carriers of repair materials. The initial studies and investigations in this area are involved with C.M. Dry [17, 18]. The method is based on the assumption that the functions of the material components can be separated into the structural and repair function. The repair substance is introduced into the concrete mix; the substance does not carry the mechanical loads, but is able to move inside the concrete and repair the damages of the microstruc- ture, without any additional, external intervention. This was innovative approach, but also brought numerous technological problems. The introduction of the repair mean on the stage of concrete production usually weakens the concrete. Moreover, this introduction should be done in such a way that its repair ability is kept during the service life of the building element.

The idea has become true by introducing the liquid repair substance, contained within the microfibres or microcapsules made of the brittle material. These microcontainers break under loading and the repair mean is released in the place of damage, then it hardens, repairing the building element.

\section{Epoxy-cement composites without hardener}

Polymer-cement concretes (PCC) are materials in which the polymer forms the separate continuous phase - co-binder. The necessity of application of the hardener as the additional component of the system is technological hindrance when producing epoxy-cement concrete or mortar. On the other hand, these materials have a number of advantages, like improved tensile and flexural strength, tightness and adhesion to various substrates $[19,20]$.

The cross-linking of an epoxy resin in the environment of Portland cement paste can proceed - to the extent dependent on accessibility of calcium hydroxide, which is a catalyst - without the presence of any hardener (Fig. 2), which can make the production of epoxy-cement composites easier.

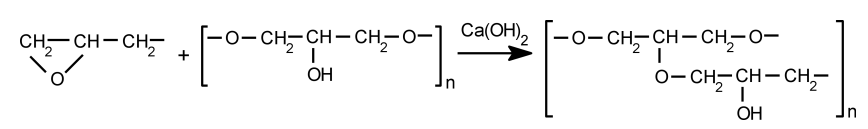

Fig. 2. Cross-linking of epoxy resin in the presence of calcium hydroxide

This phenomenon can be used for implementation of selfrepairing ability to the concrete [21]. At the polymer content of $20 \%$ in the whole binder, the degree of cross-linking of epoxy resin (when used without a hardener) is estimated to about $50 \%$ [22]. The excess of unhardened resin remains initially in the pores of the hardened cement paste. As the loadings occur, the resin is gradually released and fills the microcracks. There the resin is contacting with the calcium hydroxide, cross-linking and hardening. The microcracks are filled and tightened (Fig. 3).

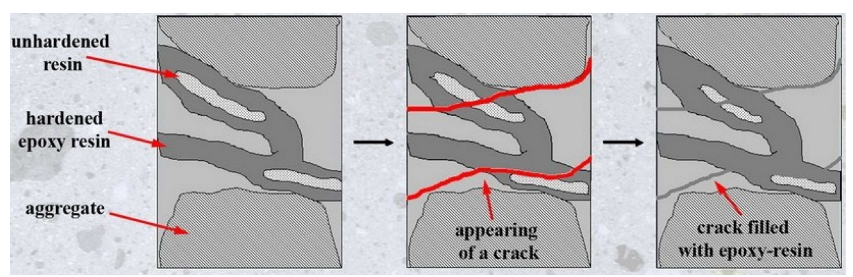

Fig. 3. Self-repairing by modification of concrete using epoxy resin without hardener

\section{Results of testing}

5.1. Method of investigation. As for now, no method of selfrepairing evaluation has been commonly accepted, nor has the 
measure of the material self-repairing ability. The original methodology of investigation of self-repairing building materials has been developed in the framework of the research project carried out by the authors, with controllable enforcing of the limited weakening of the material [23].

Several introductory research were carried out to explore various possibilities of applying load to the sample. Different types of load (bending, compression, tension), different character of load (static, dynamic) and periodicity of loading (single load, cycle of loading) were tried out. Finally, the process of a controlled weakening of a concrete sample was possible due to the precise identification of a moment when the bending load develops a crack in a sample; computer controlled testing equipment was used (Fig. 4).

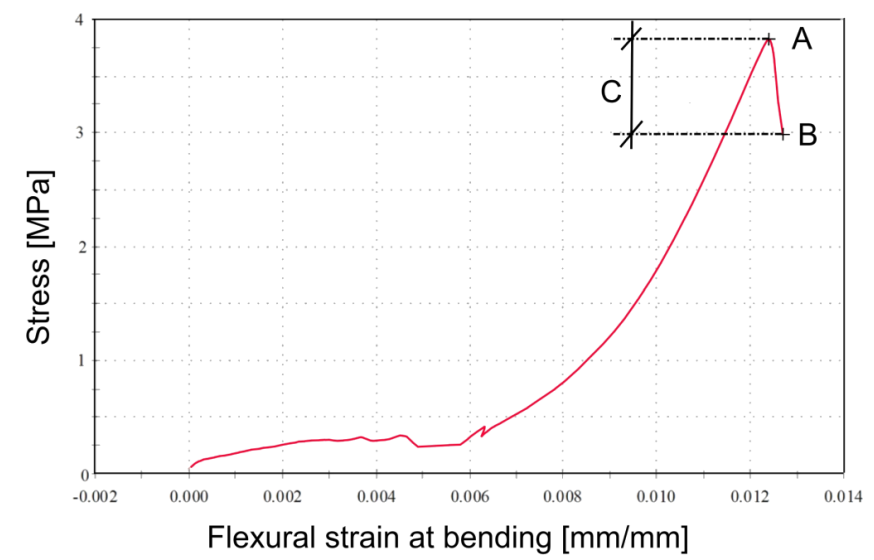

Fig. 4. Stress-strain curve obtained in bending test

Further development of a crack is connected with a decrease of applied load which results from weakening of a structure of the material being damaged. Point A on the Fig. 4 is related to the flexural strength of a sample, therefore corresponds exactly to the stress value when the first crack appears in a sample structure. Distance C corresponds to the decrease of a stress which is a consequence of crack propagation. This means that it is possible to obtain the desired crack dimension by matching an appropriate $\mathrm{C}$ value (by precise establishing the $\mathrm{B}$ value, which corresponds to the moment of stopping of the load application). After testing, the residual strength (up to the destruction of the specimen) can be determined.

In this way, the expected weakening of samples is achieved and simultaneously the flexural strength of each sample is determined, which allows to reduce the necessary number of specimens (large, due to the statistic uncertainty of results as a consequence of an inhomogeneous structure of the material).

The samples treated in this way were divided in two groups (Fig. 5). For the first one, the residual flexural strength has been determined immediately after weakening. The second group of the samples was left for a certain period of time to allow a self-repairing process to take place and after that time the flexural strength has also been determined. In the same time, the flexural strength has been determined for the reference samples, not weakened, but instead cured in a normal conditions.

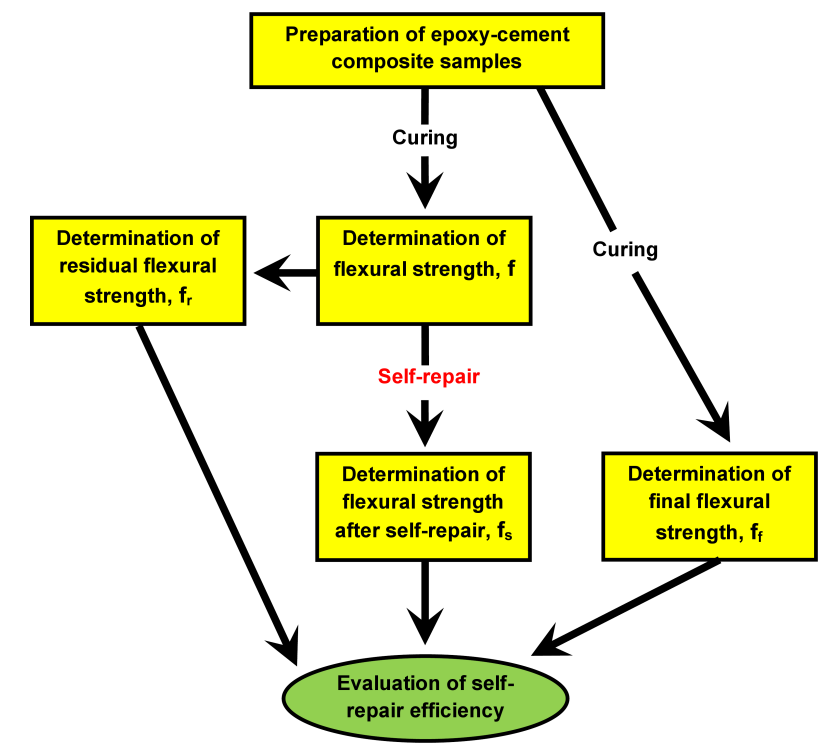

Fig. 5. Scheme of investigation of self-repairing of epoxy-cement composites

5.2. Method of evaluation of self-repairing ability of concrete. The ability to self-repair might be understood, to some extent, in the intuitive way. However, there is still a need for a quantitative measure of this feature of the building composites. Such a measure has been defined by the authors as the self-repair degree [24]. The measure is closely connected to the developed method of the investigation (Fig. 6).

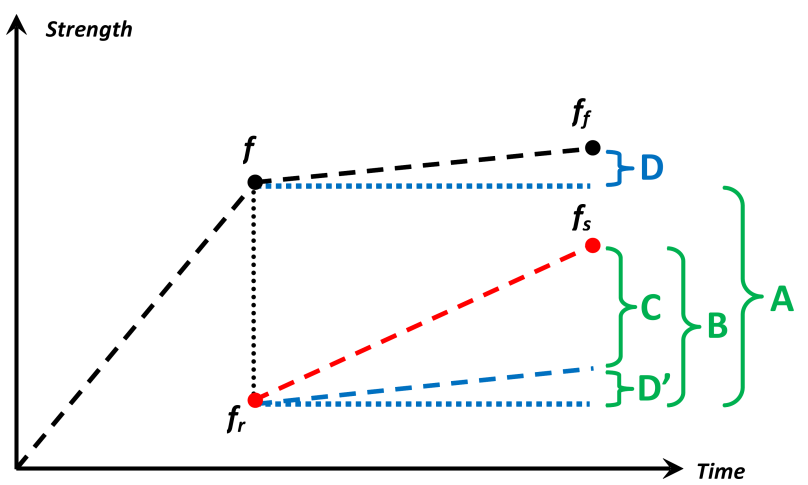

Fig. 6. Evaluation of self-repair efficiency as a result of investigation of epoxy-cement composite. Red line means increase of strength after controlled weakening of specimens, A - lowering of strength, $\mathrm{B}$ - increase of strength after self-repair, C - increase of strength connected directly to self-repair, $\mathrm{D}=\mathrm{D}^{\prime}$ - increase of strength connected to normal curing without self-repair

A self-repair degree (STS) has been accepted as the measure of self-repair efficiency. STS has been defined as the ratio of increase of strength caused by self-repair (i.e. excluding the effect of the natural curing of specimens, $C=B-D^{\prime}$ ) to decrease of strength caused by controlled weakening of the material (A):

$$
S T S=\frac{C}{A}=\frac{B-D^{\prime}}{A}=\frac{B-D}{A}=\frac{\left(f_{s}-f_{r}\right)-\left(f_{f}-f\right)}{f-f_{r}} .
$$


5.3. Results and discussion. The observations of the microstructure of the hardened mortars have been carried out using X-Ray tomography and scanning electron microscopy. The microscopic images show the specific elements of the epoxy-cement composite microstructure, for instance the continuous polymer film, visible in details (Figs. 7 and 8).
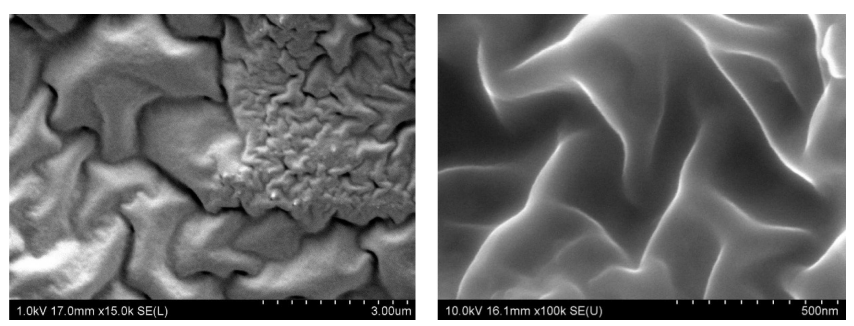

Fig. 7. Polymer film in epoxy-cement composite
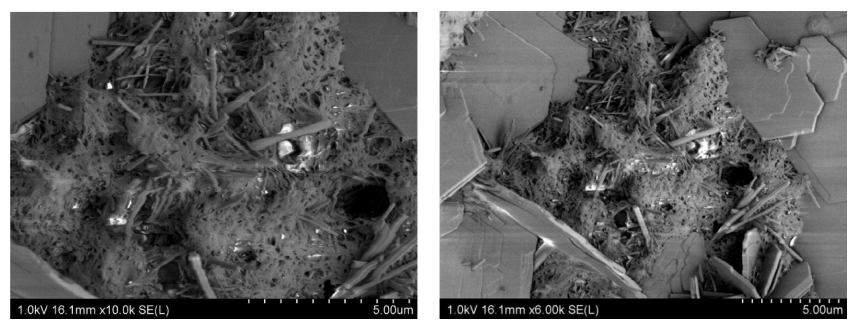

Fig. 8. Microstructure of epoxy-cement composite with visible polymer film
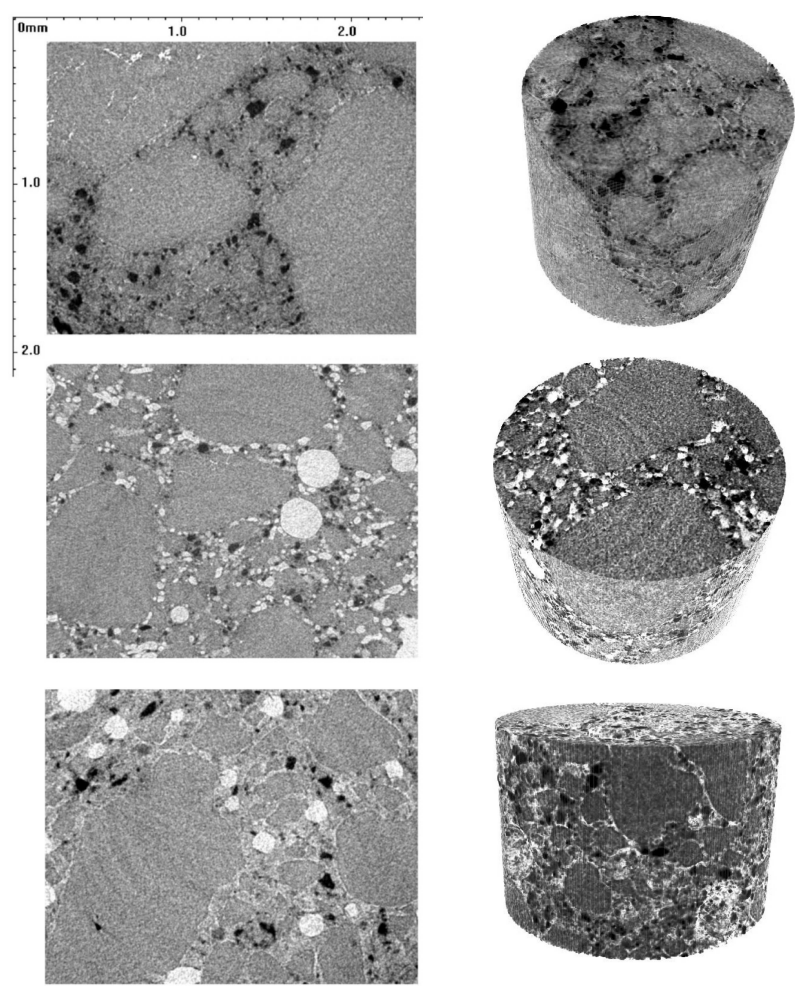

Fig. 9. Microstructure of cement composite (a), epoxy-cement composite with polymer in the form of liquid resin (b) or water emulsion (c); images from X-Ray tomography

Using of X-Ray tomography makes it possible to distinguish the areas of low density (bright fields) from those with the higher density (dark fields). This, in turn, allows to conclude about the spatial distribution of the composite components. In the case of unmodified mortar, the typical microstructure is observed, in which the aggregate grains are surrounded by the hardened cement paste (Fig. 9a). Introduction of polymer in the form of the conventional liquid resin causes formation of dispersed polymer phase, observed on the cement paste as well as in the contact zone with the aggregate (Fig. 9b). From the point of view of self-repairing, formation of the continuous layer would be of advantage - such situation can be observed when the epoxy resin is introduced in the form of the water emulsion (Fig. 9c) - the polymer film is visible, particularly in the interfacial transition zone. The reason for this difference is the way of introducing of the polymer - the conventional liquid resin is significantly viscous and thus more difficult to the uniform dispersion in the mortar (or concrete) mix. The use of water emulsion leads to more homogenous microstructure. Therefore, the emulsion of epoxy resin has been selected for using in the self-repairing tests.

The introductory tests were carried out using the epoxycement mortars with constant binder (Portland cement + epoxy resin without a hardener) to sand ratio, equal to $1: 3$ by mass. Water to cement ratio was equal to 0.5 . The water emulsion of epoxy resin was used, therefore the water contained in the emulsion was counted as the part of the mixing water. The CEM I 42.5 R cement and standard sand according to PN-EN 196-1 were also used. The investigation has been performed according to the procedure described in the point 5.1. The time of curing of the epoxy-cement mortars was 28 days in the conditions given in the European Standards (e.g. PN-EN 12190:2000 Products and systems for repair and protection of concrete structures - Test methods - Determination of compressive strength of repair mortar), i.e. 1 day covered by plastic sheet +2 days of wrapping with plastic sheet + rest of curing time in the laboratory conditions $\left(21^{\circ} \mathrm{C} \pm 2{ }^{\circ} \mathrm{C}\right.$ and $60 \% \pm 5 \% \mathrm{RH})$. For self-repairing, the specimens after weakening were left for another 28 days in the laboratory conditions. The values of self-repair degree (Fig. 10) have been calculated according to the Eq. (1).

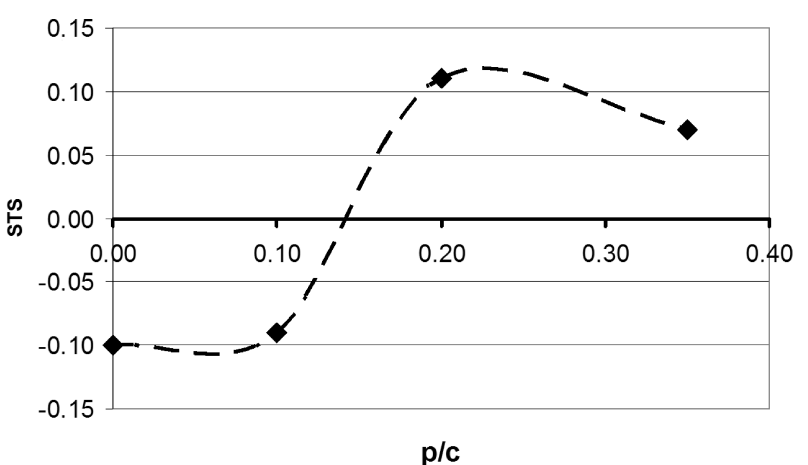

Fig. 10. Self-repair degree (STS) of epoxy-cement mortars with various contents of epoxy resin (without hardener)

The flexural strength of the epoxy-cement composite without a hardener is worse than that of unmodified mortar. The 
reason is, most likely, the presence of unhardened resin inside the material as well as the presence of emulsifiers in the water emulsion of epoxy resin. The emulsifiers hinder the effective use of water from the emulsion to the cement hydration. They may also disturb the catalytic cross-linking of the resin without a hardener. Nevertheless, the results of the introductory tests made it possible to conclude that there is such content of the epoxy resin which gives the maximum effect of self-repairing. The next step was, therefore, the material optimization of the epoxy-cement mortar towards the maximum self-repair degree.

The subject of the investigation were the epoxy-cement mortars with various content of epoxy modifier (polymer/cement ratio, $\mathrm{p} / \mathrm{c}$, was from 0.1 to 0.35 ), applied without a hardener, and various binder/aggregate ratios, b/a (from 0.33 to 0.60$)$. The research programme covered the evaluation of effectiveness of epoxy-cement composites self-repairing. The results of the testing, performed according to the statistical design of experiment, were the basis for developing of the material models of the epoxy-cement composites (Fig. 11).

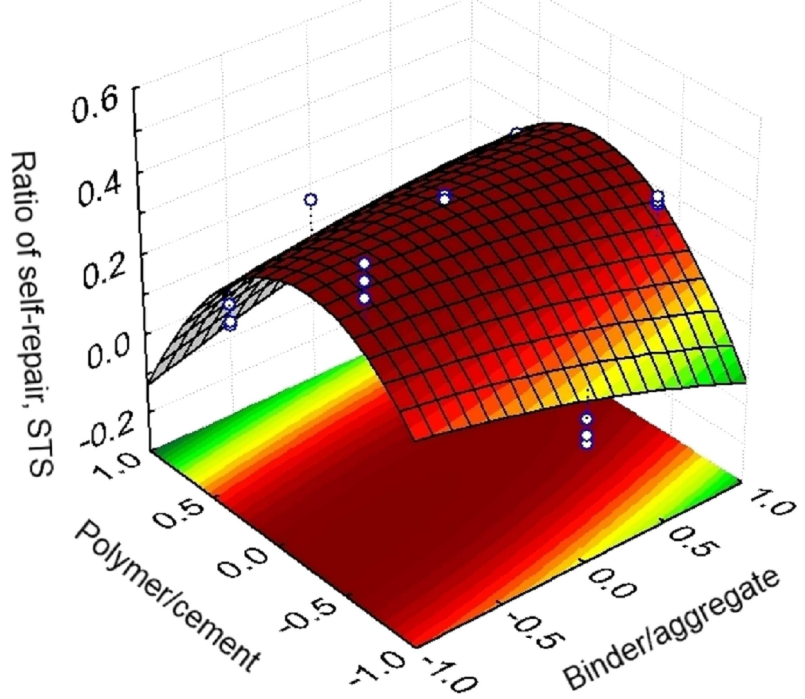

Fig. 11. Self-repair degree, STS, as a function of polymer/cement, $\mathrm{p} / \mathrm{c}$, and binder/aggregate, b/a, ratio (coded variables)

The optimization procedures including the genetic algorithm and the network method have been employed for determination of the material composition enabling the maximum effectiveness of self-repairing. The optimum values of the variables are $\mathrm{p} / \mathrm{c}_{\mathrm{opt}}=0.19$ and $\mathrm{b} / \mathrm{a}_{\mathrm{opt}}=0.34$ (real variables). The optimum content of polymer in the composite is connected to two limitations. First, some minimum amount of resin is necessary for noticeable self-repairing. However, further adding of the resin (without a hardener) decreases the mechanical features of the material and eventually the weakening of the microstructure by unhardened resin prevails over the effect of the possible self-repairing.

The verification testing has confirmed the adequacy of the developed material model. The self-repair degree, STS, of the optimized epoxy-cement composite was equal to 0.43 , with standard deviation 0.12 , while the value of STS calculated from the model was 0.41 .

The self-repair ability of the optimized epoxy-cement composite is, therefore, promising. However, comparing to unmodified mortar, some properties (flexural and compressive strength) of the epoxy-cement mortar have worsened. The other properties have changed only slightly (tensile strength) or even have improved (water absorbance, consistency of the mortar mix) (Table 1).

Table 1

Comparison of selected properties of optimized epoxy-cement mortar (without hardener) and standard cement mortar

\begin{tabular}{lcc}
\hline \hline Property & $\begin{array}{c}\text { Epoxy-cement } \\
\text { mortar } \\
\text { (without hardener) } \\
\text { optimized } \\
\text { for self-repairing }\end{array}$ & $\begin{array}{c}\text { Standard } \\
\text { cement mortar } \\
\text { (acc. to } \\
\text { PN-EN 196-1) }\end{array}$ \\
\hline Flexural strength, $\mathrm{MPa}$ & 4.2 & 6.4 \\
\hline Compressive strength, MPa & 27.2 & 47.5 \\
\hline Tensile strength, MPa & 3.4 & 3.3 \\
\hline Water absorbance, \% & 2.6 & 5.9 \\
\hline Consistency (slump), cm & 6.5 & 5.5 \\
\hline
\end{tabular}

The self-repair degree, STS, is a combined measure and its single value is calculated from the separate sets of strength values (for the samples, which were weakened or non-weakened, cured for different periods, etc.). Therefore, the statistical analysis of self-repair efficiency is difficult and requires a large number of further tests. However, because the results are most dispersed in the case of the weakened samples (the residual strength and, particularly, strength after self-repair), the limitation of their scattering is essential for the reliability of self-repairing evaluation. This conclusion is confirmed by some preliminary observations. The variation coefficient for the flexural strength after self-repair, $\mathrm{f}_{s}$, did not exceed $20 \%$, while the variation coefficient for corresponding self-repair degree, STS, sometimes reached $30 \%$ and even more. It can be noted that high variation coefficient for $\mathrm{f}_{f}$ leads to high variation coefficient for STS.

\section{Conclusions}

The results of investigations, carried out using the developed methodology, have confirmed that the cement composite can obtain the self-repairing ability by modification with the epoxy resin without the hardener. It has been demonstrated that the composition of the material enabling the maximum selfrepairing effectiveness can be established on the way of the statistical-experimental optimization. It has also been shown that self-repair degree defined in the thesis makes possible the reliable evaluation of self-repairing ability of the building material.

Polymer-cement concretes (PCC) create a large group of building composites with the granular filler. To this group are usually counted also the materials, in which the aggregate grains diameter does not exceed the size typical for the building mortars. The justification for this categorization are the properties of $\mathrm{PCC}$, in many cases very similar to those 
characteristic for the concrete [25]. The subject of investigations presented in this paper were the epoxy-cement mortars. However, taking into consideration the above explanation, the conclusions may be accepted as valid in the case of concretes, too. The verification tests of epoxy-cement concretes are now in pending.

The further intensive research in the field of self-repairing ability of the building composites are needed. The particular problem is involved with the possibility of some worsening of certain technical properties, when the epoxy resin without a hardener is introduced into the cement concrete. This can be explained by the presence of unhardened liquid resin in the composite as well as the presence of emulsifiers in the water emulsion of epoxy resin. The emulsifiers may hinder the effective use of water from the emulsion to the cement hydration and disturb the catalytic hardening of the resin. Therefore, the technological conditions should be precise for reduction of this effect. The further research directions should also cover determination of time, in which the self-repair can proceed, and possible repeating of this process.

Acknowledgements. The paper has been granted by the Ministry of Science and Academic Education, Project No. N N506 257637.

\section{REFERENCES}

[1] 1. L. Czarnecki and P.H. Emmons, Repair and Protection of Concrete Structures, Polski Cement, Kraków, 2002, (in Polish).

[2] L. Czarnecki and J. Skwara, "Repair of cracks in reinforced concrete structures by injection", Proc. Polish Conf. WPPK 1, CD-ROM (1998), (in Polish).

[3] Y. Ohama and K. Demura, "Superior properties of epoxymodified mortars and concretes without hardener", Proc. $3^{\text {rd }}$ Southern African Conf. on Polymers in Concrete and ICPIC Workshop 1, 228-239 (1997).

[4] P. Łukowski and G. Adamczewski, „Evaluation of possibility of self-repairing of epoxy-cement composite", Building Materials 5, 52-53 (2010), (in Polish).

[5] H.M. Andersson, M.W. Keller, J.S. Moore, N.R. Sottos, and S.R. White, "Self healing materials", An Alternative Approach to 20 Centuries of Materials Science 1, 19-44 (2007).

[6] P. Schiessl and N. Brauer, "Influence of autogenous healing of cracks on corrosion of reinforcement", Proc. Int. Conf. on Durability of Building Materials and Components 1, CD-ROM (1996).

[7] C. Edvardsen, "Water permeability and autogenous healing of cracks in concrete", ACI Materials J. 96, 448-455 (1999).

[8] V.C. Li and Y. En-Hua, "Self healing in concrete materials", An Alternative Approach to 20 Centuries of Materials Science 1, 161-193 (2007).
[9] T.H. Ahn and T. Kishi, "Crack self-healing behavior of cementitious composites incorporating various mineral admixtures", J. Advanced Concrete Technology, Japan Concrete Institute 2, 171-186 (2010).

[10] Y. Yang, D.M. Lepech, Y. En-Hua, and V.C. Li, "Autogenous healing of engineered cementitious composites under wet-dry cycles", Cement and Concrete Research 39, 382-390 (2009).

[11] H. Reinhardt and M. Joos, "Permeability and self-healing of cracked concrete as a function of temperature and crackwidth", Cement and Concrete Research 33, 981-985 (2003).

[12] W. Ramm and M. Biscoping, "Autogenous healing and reinforcement corrosion of waterpenetrated separation cracks in reinforced concrete", J. Nuclear Engineering and Design 179, 191-200 (1998).

[13] M. Fiertak, Structural Sealing of the Concrete Partitions. Monograph on Civil Engineering No. 271, Cracow University of Technology, Kraków, 2000, (in Polish).

[14] V.C. Li, "Engineered cementitious composites - tailored composites through micromechanical modeling", Fiber Reinforced Concrete: Present and the Future 1, 64-97 (1997).

[15] M. Wu, B. Johannesson and M. Geiker, "A review: self-healing in cementitious materials and engineered cementitious composite as a self-healing material", Construction and Building Materials 28, 571-583 (2012).

[16] V. Ramakrishnan, K. Ramesh, and S. Bang, "Bacterial concrete", Proc. SPIE Conf. 4234, 168-176 (2000).

[17] C.M. Dry, "Matrix cracking, repair and filling using active and passive modes for smart timed releases of internal chemicals", Smart Materials and Structures 3, 118-123 (1994).

[18] C.M. Dry, "Procedures developed for self-repair of polymer matrix composite materials", Composite Structures 3, 263-269 (1996).

[19] L. Czarnecki and P. Łukowski, "Polymer-cement concretes", Cement. Lime. Concrete 5, 243-258 (2010).

[20] P. Lukowski, "Polymer-cement mortars of high bond strength to the concrete substrate", Proc. $13^{\text {th }}$ Int. Congress on Polymers in Concrete ICPIC'2010 1, 665-672 (2010).

[21] T. Katsuhata, Y. Ohama, and K. Demura, "Investigation of microcracks self-repair function of polymer-modified mortars using epoxy resins without hardeners", Proc. $10^{\text {th }}$ Int. Congress on Polymers in Concrete 1, CD-ROM (2001).

[22] J.M. Butt, G.V. Topilski, V.G. Mikulski, V.V. Kozłow, and A.K. Gorban, "Investigation of interaction between epoxy resin and Portland cement", Advances in Construction and Architecture 1, 75-80 (1971), (in Russian).

[23] G. Adamczewski, "Testing of self-repairing ability of epoxycement composites", Proc. $3^{\text {rd }}$ Int. Interdisciplinary Technical Conf. Young Scientists 1, 275-278 (2010).

[24] P. Łukowski and G. Adamczewski, "Self-repairing ability of epoxy-cement concrete", Proc. Nanobrücken-Nanomechanical Testing Workshop and Hysitron User Meeting INM, CD-ROM (2010).

[25] L. Czarnecki, Resin Concretes, Arkady, Warszawa, 1982, (in Polish). 